SOF metabolite GS-3310007 were approximately 6 fold higher than in the LDV/SOF Phase 3 trials. SOF and LDV concentrations were similar to those with normal, mild or moderate RI. The most common adverse events (AEs) were fatigue (22\%), headache (22\%), and hyperkalemia (22\%). Six serious AEs were reported among 4 patients (22\%), including 2 renal events; no SAEs related to study drugs. There were no treatment-related cardiac AEs, including bradycardia, and no meaningful changes in QTc intervals or other parameters.

Conclusions Treatment with LDV/SOF (90/400 mg) for 12 weeks in genotype 1 patients with and without cirrhosis and severe renal impairment resulted in 100\% SVR12 rate. The regimen was safe and well-tolerated with no treatment discontinuations and no treatment-related SAEs. (no table selected) (No Image Selected) Co-Author Disclosure Status.

\section{IDDF2018-ABS-0112 SAFETY AND EFFICACY OF SOFOSBUVIR/ VELPATASVIR IN A GENOTYPE 1-6 HCV INFECTED POPULATION FROM SINGAPORE, MALAYSIA, THAILAND, AND VIETNAM: RESULTS FROM A PHASE 3, CLINICAL TRIAL}

${ }^{1}$ Seng Gee Lim*, ${ }^{2}$ Rosmawati Mohamed, ${ }^{3}$ Phuong Le, ${ }^{4}$ Hoi Poh Tee, ${ }^{5}$ Brian McNabb, ${ }^{5}$ Sofia Lu, Julia Lu, ${ }^{5}$ John McNally, ${ }^{5}$ Diana M Brainard, ${ }^{5} \mathrm{G}$ Mani Subramanian, ${ }^{5}$ Christina Sze Man Yip, ${ }^{5}$ Hai Cheng Huang, ${ }^{6}$ Chee Kiat Tan, ${ }^{7}$ Tawesak Tanwandee, ${ }^{8}$ Kinh Nguyen Van, ${ }^{9}$ Teerha Piratvisuth. 'Division of Gastroenterology and Hepatology, National University Hospital, Singapore; ${ }^{2}$ Department of Medicine, Faculty of Medicine, University of Malaya, Kuala Lumpur, Malaysia; ${ }^{3}$ People's Hospital 115, Ho Chi Minh City, Vietnam; ${ }^{4}$ Gastroenterology Unit, Medical Department, Hospital Tengku Ampuan Afzan, Jalan Tanah Putih, Kuantan, Pahang, Malaysia; ${ }^{5}$ Gilead Science Inc., Foster City, CA, USA; ${ }^{6}$ Department of Gastroenterology and Hepatology, Singapore General Hospital, Singapore; 'Division of Gastroenterology, Department of Medicine, Siriraj Hospital, Mahidol University, Bangkok, Thailand; ${ }^{8}$ National Hospital for Tropical Diseases, Hanoi, Vietnam; ${ }^{9}$ NKC Institute of Gastroenterology and Hepatology, Faculty of Medicine, Prince of Songkla University, Hat Yai, Songkhla, Thailand

\subsection{6/gutjnl-2018-IDDFabstracts.213}

Background The prevalence of hepatitis C virus (HCV) in Singapore, Malaysia, Thailand, and Vietnam, ranges from 1\%$3 \%$. Because of the heterogeneity of genotypes (GTs) in these countries (primarily GT1, 2, 3, and 6), there is a critical need for a pangenotypic, all oral regimen to address the burden of HCV infection. This study evaluated the efficacy and safety of SOF/VEL for 12 weeks in patients with chronic GT1-6 HCV infection.

Methods Treatment experienced and treatment naive patients with chronic GT1-6 HCV infection with no cirrhosis or with compensated cirrhosis were eligible to enrol in a single-arm, open-label trial to receive a fixed dosed combination of SOF/ VEL 400/100 mg daily for 12 weeks. Patients were recruited from 13 sites in Singapore, Malaysia, Thailand, and Vietnam. The primary efficacy endpoint was SVR12 using the CAP/ CTM HCV 2.0 assay $(\mathrm{LLOQ}=15 \mathrm{IU} / \mathrm{mL})$, and the primary safety endpoint was adverse events (AEs) leading to SOF/VEL discontinuation.

Results A total of 111 patients were enrolled and treated. Of these, 51\% were male, $14 \%$ had compensated cirrhosis, $18 \%$ were treatment-experienced, $82 \%$ had IL28B CC genotype, 20\% had GT1a, 23\% GT1b, 3\% GT2, 23\% GT3, and 31\% GT6 HCV infection. The overall SVR12 rate was 97\% (108/ 111) GT specific SVR12 rates are presented in the Table
(IDDF2018-ABS-0112 Table 1). All 3 patients who did not achieve SVR experienced a virologic relapse. All 15 cirrhotic patients, including 8 with GT3 HCV infection achieved SVR12. Virology data will be presented. There were no discontinuations due to AEs. There were 50 patients (45\%) who experienced any adverse events (AEs), with no AEs occurring in $>10 \%$ of patients. No serious or severe AEs were assessed by the investigator as related to study drug and there were no deaths.

\begin{tabular}{|c|c|c|c|c|c|c|c|c|}
\hline \multirow[t]{2}{*}{$\mathrm{N}(\%)$} & Total & GT1a & GT1b & GT1 & GT2 & GT3 & GT6 & Indeterminate ${ }^{\mathrm{a}}$ \\
\hline & $\mathrm{n}=111$ & $n=22$ & $n=25$ & $\begin{array}{l}\text { (total) } \\
n=47\end{array}$ & $n=3$ & $\mathrm{n}=25$ & $n=34$ & $n=2$ \\
\hline SVR12 & $\begin{array}{c}108 / 111 \\
(97)\end{array}$ & $\begin{array}{l}22 / 22 \\
(100)\end{array}$ & $\begin{array}{l}25 / 25 \\
(100)\end{array}$ & $\begin{array}{l}47 / 47 \\
(100)\end{array}$ & $\begin{array}{c}3 / 3 \\
(100)\end{array}$ & $\begin{array}{c}23 / 25 \\
(92)\end{array}$ & $\begin{array}{c}33 / 34 \\
(97)\end{array}$ & $2 / 2(100)$ \\
\hline
\end{tabular}

Conclusions Treatment with the single tablet, pangenotypic regimen of SOF/VEL for 12 weeks was highly effective and well tolerated in a GT 1,2,3, and $6 \mathrm{HCV}$ infected population with and without cirrhosis from Singapore, Malaysia, Thailand, and Vietnam. *Seng Gee Lim is the presenter of the abstract.

\section{IDDF2018-ABS-0114 SOF/VEL/VOX RESULTS IN HIGH SVR12 RATES WHEN ADMINISTERED FOR 12 WEEKS IN DAA-EXPERIENCED PATIENTS OR FOR 8 WEEKS IN DAA-NAIVE PATIENTS: AN INTEGRATED ANALYSIS OF THE POLARIS-1, POLARIS-2, POLARIS-3 AND POLARIS-4 STUDIES}

${ }^{1}$ Stuart K Roberts* ${ }^{2}$ Curtis L Cooper, ${ }^{3}$ Eric Lawitz, ${ }^{4} \mathrm{~K}$ Rajender Reddy, ${ }^{5}$ Alex J Thompson, ${ }^{6}$ Stefan Zeuzem, ${ }^{7}$ Ira M Jacobson, ${ }^{8}$ Peter Ruane, ${ }^{9}$ Robert H Hyland, ${ }^{9}$ Luisa M Stamm, ${ }^{9}$ Lingling Han, ${ }^{9}$ Diana M Brainard, ${ }^{9}$ Christina SM Yip, ${ }^{9} \mathrm{HC}$ Huang, ${ }^{10}$ Norbert Brau, ${ }^{11}$ Tarik Asselah, ${ }^{12}$ Bernard E Willems, ${ }^{13}$ Steven Flamm, ${ }^{14}$ Marc Bourliere, ${ }^{15} \mathrm{Graham}$ R Foster, ${ }^{16}$ Edward J Gane, ${ }^{17}$ Michael Manns, ${ }^{18}$ Stuart C Gordon, ${ }^{19}$ Kris Kowdley. ${ }^{1}$ Alfred Hospital, Melbourne, Australia; ${ }^{2}$ Ottawa Hospital Research Institute, Ottawa, Canada; ${ }^{3}$ Texas Liver Institute, University of Texas Health Science Center, San Antonio, USA; ${ }^{4}$ University of Pennsylvania, Philadelphia, USA; ${ }^{5}$ Department of Gastroenterology, St. Vincent's Hospital, Melbourne, Australia; ${ }^{6} J$ Johann Wolfgang Goethe University Medical Center, Frankfurt, Germany; ' Department of Medicine, Mount Sinai Beth Israel, New York, USA; ${ }^{8}$ Ruane Medical and Liver Health Institute, Los Angeles, USA; ${ }^{9}$ Gilead Sciences, Inc., Foster City, USA; ${ }^{10}$ Mount Sinai School of Medicine, New York, USA; ${ }^{11}$ Service $d^{\prime}$ Hepatologie, Hopital Beaujon, Universite Paris Diderot, Clichy, France; ${ }^{12}$ Northwestern University, Chicago, USA; ${ }^{13}$ Hospital Saint Joseph, Marseille, France; ${ }^{14}$ Royal London Hospital, London, UK; ${ }^{15}$ Auckland Clinical Studies, Auckland, New Zealand; ${ }^{16}$ Department of Gastroenterology, Hepatology and Endocrinology, Hannover Medical School, Hannover, Germany; ${ }^{17}$ Henry Ford Health System, Detroit, USA; ${ }^{18}$ Swedish Medical Center, Seattle, USA; ${ }^{19}$ Swedish Medical Center, Seattle, United States

\subsection{6/gutjnl-2018-IDDFabstracts.214}

Background The once-daily fixed-dose combination tablet of sofosbuvir/velpatasvir/voxilaprevir(SOF/VEL/VOX) was evaluated for the treatment of genotype 1-6 HCV infection in four Phase 3 studies in direct-acting antiviral (DAA)-experienced (POLARIS-1 and POLARIS-4) and DAA-naive 
(POLARIS-2 and POLARIS-3) patients with and without compensated cirrhosis. DAA-experienced patients received treatment for 12 weeks, and DAA-naive patients received treatment for 8 weeks. Overall SVR12 rates were $>95 \%$ across all the studies. This post-hoc analysis assesses efficacy in patients with and without traditional negative predictors of response.

Methods This was a retrospective analysis of data from 1056 patients treated with SOF/VEL/VOX in the Phase 3 studies. Presence of cirrhosis was determined by histology, Fibrotest/ APRI, or Fibroscan. Viral load and other clinical and laboratory assessments were determined prior to treatment with SOF/VEL/VOX. Prior treatment records were source verified, and race was self-reported by the patient to the investigator.

Results Overall, 38\% of patients had cirrhosis, $70 \%$ had HCV RNA $\geq 800,000 \mathrm{IU} / \mathrm{mL}, 59 \%$ of the DAA-experienced patients had received an NS5A inhibitor-containing regimen, 20\% of the DAA-naive patients had prior treatment failure with pegylated interferon +ribavirin, $12 \%$ were $\geq 65$ years old and $10 \%$ were black.

Conclusions The POLARIS-1, POLARIS-2, POLARIS-3, and POLARIS-4 studies enrolled a diverse patient population that included a significant number of patients with historically negative predictors of response including cirrhosis and prior exposure to DAA-containing regimens. High SVR12 rates for the ribavirin-free regimen of SOF/VEL/VOX were achieved across subgroups.

\begin{tabular}{|c|c|c|}
\hline Overall & $430 / 445$ (97) & $582 / 611$ (95) \\
\hline Cirrhosis & $194 / 205$ (95) & $188 / 200$ (94) \\
\hline HCV RNA $\geq 800 \mathrm{~K}$ & $317 / 326$ (97) & $392 / 416$ (94) \\
\hline Prior PEG+RBV & - & $114 / 124$ (92) \\
\hline Age $>65$ & 73/74 (99) & 55/57 (96) \\
\hline Black & $50 / 54(93)$ & $43 / 48(90)$ \\
\hline
\end{tabular}

\section{IDDF2018-ABS-0120 THE EFFECT OF OMEGA-3 FATTY ACID SUPPLEMENTATION ON PAEDIATRIC NON- ALCOHOLIC FATTY LIVER DISEASE: A META-ANALYSIS OF RANDOMISED CONTROLLED TRIALS}

${ }^{1}$ Martin MH Wong*, ${ }^{2}$ Kenneth Lo, ${ }^{3}$ Wilson Tam. ${ }^{1}$ The Nethersole School of Nursing, The Chinese University of Hong Kong, Hong Kong; ${ }^{2}$ School of Public Health and Primary Care, The Chinese University of Hong Kong, Hong Kong; ${ }^{3}$ Alice Lee Centre for Nursing Studies, Yong Loo Lin School of Medicine, National University of Singapore, Singapore

\subsection{6/gutjint-2018-IDDFabstracts.215}

Background Paediatric non-alcoholic fatty liver disease (NAFLD) is an obesity-related disease with growing prevalence worldwide. While current guidelines recommend lifestyle modification, omega-3 supplementation has been suggested as an emerging therapeutic option. Despite supporting evidence demonstrated among adult NAFLD patients, similar meta-analysis among the paediatric population is lacking. The present review aimed to evaluate the efficacy of omega-3 supplementation among children with NAFLD.

Methods A systematic review of all randomised controlled trials (RCT) on NAFLD patients aged 18 or under was conducted using 16 multiple databases including Medline, EMBASE and Cochrane Central Register of Controlled Trials. PRISMA guideline was followed. The search was conducted by two independent reviewers, with discrepancies resolved by the third reviewer. Outcomes were categorised into anthropometric (e.g. body mass index), cardio-metabolic (e.g. triglycerides and insulin resistance) and hepatic outcomes (e.g. liver enzymes). Using mean differences (MD), meta-analysis was conducted on outcomes reported in more than one study using a random-effect model. If only median and inter-quartile ranges were provided, they were transformed into mean and standard deviation before meta-analysis. Heterogeneity was examined using $\mathrm{I}^{2}$ statistics. The Cochrane Collaboration's tool for assessing the risk of bias was used to examine the quality of the included studies.

Results After screening 2962 papers, six papers with 326 subjects from five RCT were included in the present review. The included studies were conducted in Canada, Italy, Poland and Turkey. Omega-3 supplementation significantly increased body mass index $\mathrm{z}$-score $(\mathrm{MD}=0.09)$, reduced the levels of serum triglycerides $(\mathrm{MD}=-9.52)$ and alanine aminotransferase (ALT) $(\mathrm{MD}=-12.04)$, and improved insulin sensitivity (MD of the homeostasis model assessment of insulin resistance $=-0.49$ ). Heterogeneity was low (below 30\%) across various outcomes, and the study quality was generally high, with three papers having low risks of bias in all six domains.

Conclusions To conclude, omega-3 supplementation reduced serum triglycerides and ALT, and improved insulin sensitivity among children with NAFLD. It may act as a potential supplementation for paediatric NAFLD patients. But more trials should be conducted, particularly in Asian regions.

\section{IDDF2018-ABS-0124 IDENTIFICATION OF MIR-148A IN PLASMA AS A POTENTIAL NONINVASIVE BIOMARKER FOR HEPATOCELLULAR CARCINOMA}

${ }^{1}$ Juqiang Han*, ${ }^{2}$ Jiarui Li, ${ }^{3}$ Wenpeng Liu, ${ }^{2}$ Jiguang Liang, ${ }^{4}$ Zhigang Huang, ${ }^{1}$ Shuai Wang, ${ }^{3}$ Caiyan Zhao, ${ }^{5}$ Xiaojie Xu, ${ }^{5}$ Qinong Ye. ${ }^{1}$ Department of Liver Disease, PLA Army General Hospital, China; ${ }^{2}$ Department of Interventional Radiography, The First Hospital of Jilin University, China; ${ }^{3}$ Department of Infectious disease, The Third Hospital of Hebei Medical University, China; ${ }^{4}$ Department of Epidemiology, Guangdong Medical University, China; ${ }^{5}$ Department of Medical Molecular Biology, Beijing Institute of Biotechnology, China

\subsection{6/gutjnl-2018-IDDFabstracts.216}

Background Detection of microRNA (miRNA) aberrations in the peripheral plasma is a new approach for hepatocellular carcinoma (HCC) screening. The aim of this study was to characterise miR-148a in the peripheral plasma as a non-invasive biomarker for diagnosis of HCC.

Methods Plasma-based miR148a analysis was performed on 346 plasma samples, including 155 HCCs, 96 liver cirrhosis and 95 healthy controls by using quantitative Real-Time PCR (qRT-PCR). Subsequently, plasma-based miR148a levels were validated in 97 pairs of HCC followed-up after removal of 